\title{
Transumbilical Laparoscopic Assisted Single Port Appendectomy (Hybrid Appendectomy) in Children
}

\author{
Sang Hyup Han, M.D., Jin Won Lee, M.D., Jeong Hee Han, M.D., Hae Sung Kim, M.D., Byoung Yoon Ryu, M.D. \\ Department of Surgery, College of Medicine, Hallym University, Chuncheon, Korea
}

Purpose: Laparoscopic appendectomy isused as a three port technique for appendectomy. In children, single port laparoscopic appendectomy is difficult because they have a small peritoneal cavity for manipulation of laparoscopic instruments. We performed transumbilical laparoscopic assisted single port appendectomy (hybrid appendectomy) in children.

Methods: From March 2010 to July 2012, we performed transumbilical laparoscopic assisted single port appendectomy in 53 children. We made a vertical incision to the umbilicus approximately $1.5 \mathrm{~cm}$, and a wound retractor (Applied Medical Resources Co., Ltd., Rancho Santa Margarita) was placed in the umbilical incision, and appendix exteriorized the extraperitoneum through the wound retractor. Appendectomy was performed conventionally. We had no conversion cases for laparotomy.

\section{INTRODUCTION}

Laparoscopic appendectomy has been used progressively as an emergency procedure. Since laparoscopic appendectomy described by Semm ${ }^{1}$ in 1983, it has improved technically as two-port technique, hybrid approaches, needlescope appendectomy. ${ }^{2}$ Laparoscopic appendectomy through a single umbilical incision has been reported as early as 1992 in adults $^{3}$ and 1999 in children $^{4}$ using a combined intra- and extracorporeal method. This technique comprises identifying and mobilizing the appendix laparoscopically with a working, multichannel laparoscope through the umbilicus but performing the actual appendectomy extracorporeally. Recently, single-incision laparoscopic appendectomy in children has regained popularity ${ }^{5}$ and has evolved to a purely intracorporeal approach. ${ }^{5-7}$

Despite the potential benefits to patients, the single site approach has potential risk. The tight approximation of instrument restricts the surgeons' hand to a narrow range of motion. The parallel alignment of these instruments limits triangulation

Received August 24, 2012, Revised 1st, September 11, 2012; 2nd, September 28, 2012, Accepted October 14, 2012

※ Corresponding author : Byoung Yoon Ryu

Department of Surgery, College of Medicine, Hallym University,

153, Gyo-dong, Chuncheon 200-704, Korea

Tel : +82-33-252-9970, 5216, Fax : +82-33-243-6413

E-mail : byryu@hallym.or.kr

http://dx.doi.org/10.7602/jmis.2012.15.4.145
Results: A total of 53 patients, 29 females and 24 males, with a mean age of $8.5 \pm 2.0$ years were enrolled in this retrospective study. The mean operative time was $29.4 \pm 9.4$ minutes. There was no occurrence of complication or mortality. BMI was $17.8 \pm 4.9 \mathrm{~kg} / \mathrm{m}^{2}$. And mean hospital stay was $3.2 \pm 1.0$ days.

Conclusion: In children, transumbilical single port laparoscopic appendectomy is technically difficult because they have a small peritoneal cavity. However, transumbilical laparoscopic assisted single port appendectomy (hybrid appendectomy) appearsto be a safe and effective technique for use in children, which allows for achievement of nearly scarless surgery.

Key words: Appendectomy, Laparoscopy, Children which is a founding principle of safe and effective laparoscopic surgery. Furthermore, inline placement of the scope narrows the visual field and forces the field of view to be dependent and limited by the movements of the instruments. ${ }^{8}$ In children, single port laparoscopic appendectomy is difficult because they have small peritoneal cavity for manipulation of the laparoscopic instruments. So we performed transumbilical laparoscopic assisted single port appendectomy more easily.

\section{MATERIALS AND METHODS}

From December 2008, we performed 53 cases transumbilical laparoscopic assisted single port appendectomy in children. The appendicitis was diagnosed with physical examination, abdominal sonography, and abdominal CT and periappendiceal abscess was excluded for laparoscopic surgery. They received therapeutic antibiotics intravenously at the induction of an anesthesia, and after surgery. All these procedures were performed by an experienced single surgeon in single site appendectomy.

\section{1) Operative technique}

The surgeon stood on the left side and the assistant on the right side of the patient while the monitor was placed on the right side. Under the general anesthesia, a $1.5 \mathrm{~cm}$ vertical incision to the umbilicus was made. After a window have been made, a small sized wound retractor (Alexis, Applied Medical resources Co., Ltd., rancho Santa Margarita) was inserted 


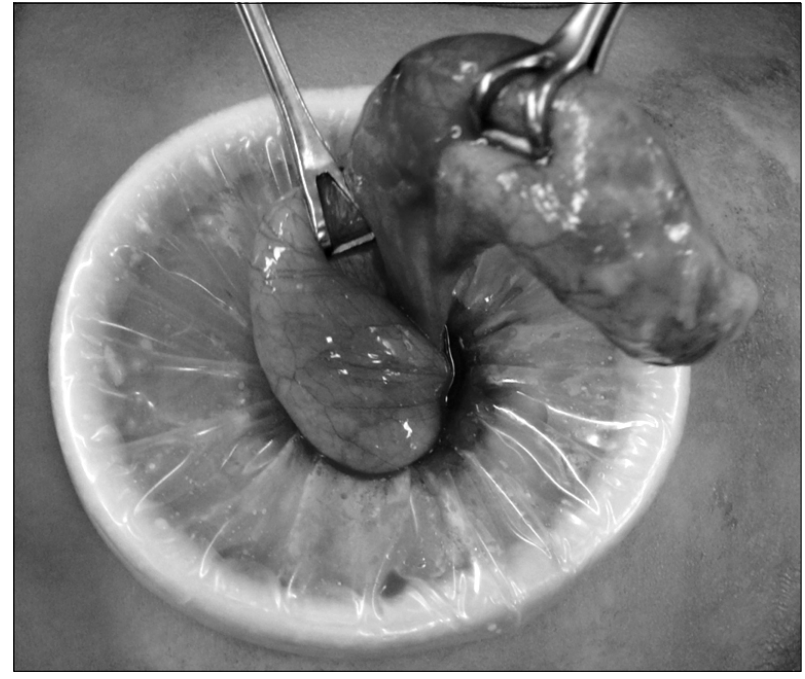

Fig. 1. The appendix was exterorized through the wound retractor.

through the incision and rolled over to cover and stretch it. The glove was then attached on the wound retractor ring, which was rolled over it to provide a seal. After pneumoperitoneum was established, a $5 \mathrm{~mm} 30$ degree videoscope was inserted into the third finger of the glove and the other trocar inserted to other finger of the glove. On videscope, appendix was identified and exteriorized through the wound retractor (Fig. 1). Attached glove was removed from the wound retractor and then appendectomy was performed conventionally. Umbilical skin incision was approximated by subcutaneous sutures and compressive dressing was done with peanut ball sponge (Fig. 2).

\section{RESULTS}

This study included 29 females and 24 males with a mean age of $8.5 \pm 2.0$ years. The mean BMI was $17.9 \pm 4.9 \mathrm{~kg} / \mathrm{m}^{2}$ (Height; 1.26 1.49 m, Weight; 16 59 kg). The procedure was performed on 53 patients, without alteration of conventional appendectomy, appendectomy with drainage was 5 cases. The mean operative time was $29.4 \pm 9.4$ minutes. Postoperatively oral liquids were started within 10 hours and a soft diet within 24 hours. Pathologic findings were commonly acute catarrhal appendicitis and acute suppurative appendicitis. All patients was not developed postoperative complication. The median hospital stay was $3.2 \pm 1.0$ days. The postoperative scar was hidden in the wrinkle of the umbilicus.

\section{DISCUSSION}

The laparoscopic appendectomy started by Semm in 1983,

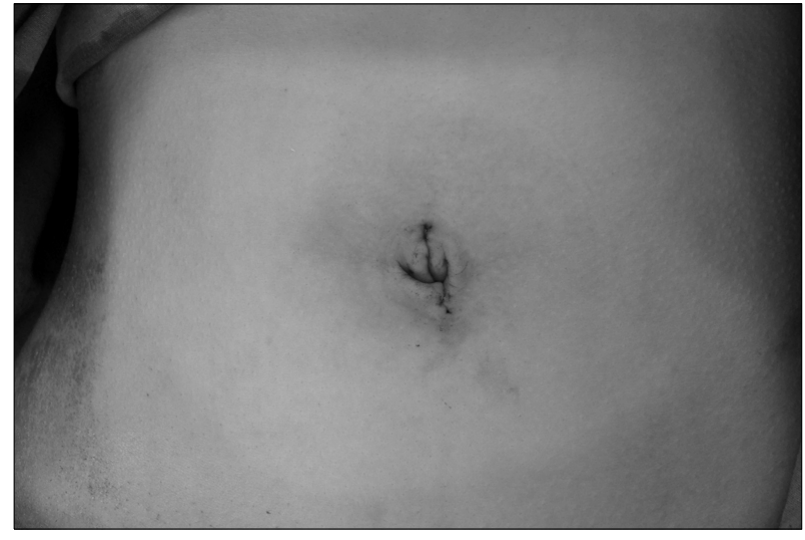

Fig. 2. Postoperative wound.

offering a promising alternative1. In 1992, Gilchrist et al. ${ }^{9}$ described first series of laparoscopic appendectomy in pediatric patients. Metaanalysis of 23 studies that included 6,477 pediatric patients and compared operative data and outcomes of open and laparoscopic methods, gave same evidence about validity of procedures. A reduced incidence of wound infection and ileus rate are major advantages of laparoscopy. Shorter postoperative hospital stay after laparoscopy was also reported. ${ }^{9}$

The single port transumbilical laparoscopic assisted appendectomy (TULAA) technique, as described by Esposito, ${ }^{10}$ provided the additional advantages of the combined approach. An idea to apply principles and strategies of laparoscopy and compile advantage resulted in development laparoscopic assisted techniques. These techniques consisted of two parts; intra-abdominal mobilization and extracorporeal removal of the appendix. The procedure is rarely used because all intra-abdominal dissection must be done with an instrument that passes through the working channel of the telescope. For this reason, special equipment is necessary, including a telescope with working channel and long shift instruments.

The hybrid technique, which combines laparoscopy with standard open techniques, the appendix is pulled through the umbilicus in children or a right lower quadrant incision in adults for performance of a traditional open appendectomy extracorporeally. ${ }^{11,12}$ The hybrid technique in which the appendix is pulled through the single incision in the umbilicus is possible only in the pediatric population due to the close proximity of the appendix and the umbilicus. ${ }^{13}$ The umbilicus is located in the thinner area of the abdominal wall, and this condition makes the port introduction easier as well as motion of the instrument in all directions. ${ }^{14}$

In pediatric surgery, hybrid and single-port assisted laparoscopic appendectomies have been popularized because of 
the surgeon's ability to grasp the appendix and exteriorize it through the umbilical port to perform an appendectomy extracorporeally. ${ }^{11,15}$

Extracorporeal ligation of the mesoappendix and reduced time needs for port access and closure seems to be responsible for difference between TULAA and laparoscopic appendectomy. ${ }^{16}$ We can easily perform appendectomy in children.

Exteriorization of an intraabdominal drain through the umbilical wound was not considered due to an increased risk of cutaneous infection at this level and the possibility of incisional hernia. Moreover, insertion of a 5-mm trocar at the hypogastric level may help in safe handling of intestinal loops and would facilitate placement of a drain through the cutaneous orifice. ${ }^{14}$ We placed J-P drain in 5 cases to the right lower abdominal area through the umbilical incision.

Different studies describing transumbilical appendectomy have been published in the literature. In 1996, Kala et al. ${ }^{17}$ performed the procedure through one umbilical port with extracorporeal section of the appendicular structures. A large variety of umbilical procedures with or without exteriorization of the appendix have been reported in clinical series of pediatric patients. ${ }^{10,18,19}$ A group at the University of Naples ${ }^{18}$ described transumbilical appendectomy using a single trocar and a flexible laparoscope with extracorporeal section of appendicular structures. However, the authors indicate the need to use supplemental trocars or to perform a small laparotomy in McBurney's point when appendicular inflammations or intraperitoneal adherences in the right iliac fosse are found. ${ }^{14}$

We used a transumbilical single port that consisted of a wound retractor and a surgical glove. This devices has several merits, it can prevent subcutaneous emphysema, as well as port-site infection and bleeding, due to the tamponade effect of the wound retractor. During surgery, rolling up the ring of the wound retractor with surgical glove is important to prevent the leakage of insufflated gas, and to prevent contamination of the wound.

All our patients could be operated by transumbilical laparoscopic assisted appendectomy technique without conversions or additional ports and they had an uneventful recovery. If necessary, the surgeon can convert laparoendoscopic single-site surgery (LESS) into a conventional laparoscopic procedure by adding another trocar while preserving safety for the patient. ${ }^{14}$

\section{CONCLUSION}

In conclusion, we report our experiences of transumbilical laparoscopic assisted appendectomy (hybrid appendectomy) in
53 patients. All patients were operated successfully without any complications. It could be an appropriate minimally invasive technique for appendectomy in children.

\section{REFERENCES}

1) Semm K. Endoscopic appendectomy. Endoscopy 1983;15:59-64.

2) Sajid MS, Khan MA, Cheek E, Baig MK, Needlescopic versus laparoscopic appendectomy: a systemic review. Can J Surg 2009;52:129-134.

3) Pelosi MA, Pelosi MA 3rd. Laparoscopic appendectomy using a single umbilical puncture (minilaparoscopy). J Reprod Med 1992;37:588-594.

4) Valla J, Ordorica-Flores RM, Steyaert H, et al. Umbilical one-puncture laparoscopic-assisted appendectomy in children. Surg Endosc 1999;13:83-85.

5) Ponsky TA, Diluciano J, Chwals W, Parry R, Boulanger S. Early experience with single-port laparoscopic surgery in children. J Laparoendosc Adv Surg Tech A 2009;19:551-553.

6) Rothenberg SS, Shipman K, Yoder S. Experience with modified single-port laparoscopic procedures in children. $\mathrm{J}$ Laparoendosc Adv Surg Tech A 2009;19:695-698.

7) Dutta S. Early experience with single incision laparoscopic surgery: eliminating the scar from abdominal operations. J Pediatr Surg 2009;44:1741-1745.

8) Garey CL, Laituri CA, Ostlie DJ, St Peter SD. A review of single site minimally invasive surgery in infants and children. Pediatr Surg Int 2010;26:451-456.

9) Gilchrist BF, Lobe TE, Schropp KP, et al. Is there a role for laparoscopic appendectomy in pediatric surgery. J Pediatr Surg 1992;27:209-212.

10) Esposito C. One-trocar appendectomy in pediatric surgery. Surg Endosc 1998;12:177-178.

11) Valla J, Ordorica-Flores RM, Steyaert H, et al. Umbilical one-puncture laparoscopic-assisted appendectomy in children. Surg Endosc 1999;13:83-85.

12) D'Alessio A, Piro E, Tadini B, Beretta F. One-trocar transumbilical laparoscopic-assisted appendectomy in children: our experience. Eur J Pediatr Surg 2002;12:24-27.

13) Roberts KE. True single-port appendectomy: first experience with the "puppeteer technique". Surg Endosc 2009;23: 1825-1830.

14) Vidal $O$, Valentini $M$, Ginestà $C$, et al. Laparoendoscopic single-site surgery appendectomy. Surg Endosc 2010;24:686-691.

15) Meyer A, Preuss M, Roesler S, Lainka M, Omlor G. Transumbilical laparoscopic-assisted "one-trocar" appendectomyTULAA-as an alternative operation method in the treatment of appendicitis. Zentralbl Chir 2004;129:391-395.

16) Visnjic S. Transumbilical laparoscopically assisted appendectomy in children: high-tech low-budget surgery. Surg Endosc 2008;22:1667-1671. 
148 Journal of Minimally Invasive Surgery Vol. 15. No. 4, 2012

17) Kala Z, Hanke I, Newmann C. A modified technique in laparoscopy-assisted appendectomy, a transumbilical approach through a single port. Rozhl Chir 1996;75:15-18.

18) Rispoli G, Armellino MF, Esposito C. One-trocar appendectomy.
Surg Endosc 2002;16:833-835.

19) Hong TH, You YK, Lee KH. Transumbilical single-port laparoscopic cholecystectomy: scarless cholecystectomy. Surg Endosc 2009;23:1393-1397. 